\title{
Reducing Death Anxiety: The Impact of Advanced Care Planning
}

\author{
Kendra Hollenbeck ${ }^{1}$, Tom Gutwein ${ }^{2}$
}

${ }^{1}$ Indiana University School of Medicine; ${ }^{2}$ Parkview Health Emergency Medicine

Background/Objective: Death anxiety affects multiple areas of patient's physical and mental health. Reducing this phenomenon has the potential to improve quality of end of life (EOL). The effects of spirituality, age, self-esteem, biological sex, presence of symptoms and level of isolation have been previously studied. Advanced care planning (ACP), a simple intervention, which has known positive impacts on quality of EOL by decreasing healthcare costs and undesired hospitalizations, however, only $33 \%$ of the population has completed it. This study will examine the correlation between death anxiety and ACP. We hypothesize that there will be a statistically significant lower level of death anxiety in participants with ACP.

Methods: The study population will include patients presenting to the Parkview Regional Medical Center ED who are $\geq 65$, with a sample size of 1,000 . We will use a translated and adapted version of the 17 question Likert-type Scale of Death Anxiety; developed and validated by Cai et al. in 2017. It will be presented via REDCap and will include electronic signature of informed consent. Finally, we will include questions about ACP and advanced directives. Participants will also complete a basic demographic survey to account for confounding factors. Analysis will include a Mann Whitney test, univariate and multivariate regressions.

Results: This project resulted in a IRB submission drafts of a study protocol, informed consent, SDA survey and REDCap data collection tool. The researchers are seeking Parkview HealthIRB approval in Fall 2020, with an anticipated study start date of Spring 2021.

Impact: This project has the potential to improve quality of life by decreasing death anxiety and resulting negative stress reactions. Interventions for decreasing death anxiety have not been extensively studied so directions for future research are broad and could include impacts of ACP in varying ages and contexts outside of the ED. 